\title{
The Efficacy and Safety of Plant Oil Mixtures in the Treatment of Xerosis with Pruritus in Elderly People: Randomized Double Blind Controlled Trial \\ Yulia Farida Yahya, Dimas Ega Wijaya Putra, Cyntya Sari Sovianti, Damai Trislinawati, Tiar Marina Octyvani*, Nuri Rifiati**
}

*Dep. Dermatology and Venereology,**Dep Internal Medicine

Medical Faculty University of Sriwijaya, Palembang- Indonesia

\section{A R T I C L E I N F O}

Keywords:

Plant Oil Mixtures (Calendula, Lavender, Chamomile, Rosemary)

$\mathrm{VCO}$

Clinical Dry Score

Pruritus Severity

Skin Surface Lipid

Skin Sebum Level

\section{Corresponding author: \\ Tiar Marina Octyvani \\ E-mail address: \\ tiarmarinaoctyvani@gmail.com}

All authors have reviewed and approved the final version of the manuscript.

https://doi.org/10.32539/bsm.v5i3.206

\begin{abstract}
A B S T R A C T
Background: Xerosis skin and pruritus is the most commonly found in elderly with symptoms mild to severe recurrent pruritus, the skin looks rough, scaly, fissured or cracked caused defects in the epidermal barrier function, increase co-morbidity as chronic infections and ulcers that are difficult to heal can decrease the quality of life. Virgin Coconut Oil (VCO) has been traditionally used for people in tropical region as a moisturizer and anti-bacterial effect. In recent study that essential plant oil mixture have effect synergistically as anti inflammatory, anti pruritus, promoting wound healing may act promoting barrier skin and therapeutic. Objective: Efficacy and safety of plant oil mixtures as therapeutic and maintenance moisturizer.for Xerosis and pruritus in elderly Method:A randomized controlled trial was involved 61 elderly from nursing home and geriatric clinic in Moh. Hoesin General Hospital. They were randomized into 2 groups: plant oil mixtures $(n=30)$ and Virgin Coconut oil $(\mathrm{VCO})(\mathrm{n}=31)$, applied on the volar legs twice a day for 4 weeks. Outcomes were measured at baseline and weekly, week 2 and 4.To measure of clinical dry score used ODSS; pruritus severity and side effect used VAS; for skin hydration used corneometer CM825and for sebum level used sebumeter SM 810. Results:Clinical dry score, pruritus severity of plants oil mixtures group were significantly decreased than VCO at week 2 and 4 (p 0.000). Skin hydration and sebum level of plants oil mixture group were significantly increased than VCO at week 2 and 4 (p 0.000).Clinical dry score, pruritus severity, skin hydration, andskin sebum levels of plant oil mixtures were significantly better than VCO at week 4 (p 0.000). There is no side effects on both of plant oil mixtures and VCO groups Conclusion: Plant oil mixtures add pureCelin oil showed better efficacy as a therapeutin and maintanance moisturizer for xerosis and pruritus in elderly. Both of plant oil mixtures and VCO can be tolerated and safe to use.
\end{abstract}

\section{Introduction}

Xerosis cutis or dry skin known as eczema craquele, dysidrotic eczema or asteatotic eczema is a common problem, especially the elderly, with clinical manifestations in the form of thin skin, atrophy, rough, dry, scaly, uneven skin color, wrinkle, smooth and deep fissure, dull, accompanied by mild to severe pruritus, recurrence The prevalence of xerosis in the elderly ranges from $29.5-85 \%$. The causes of xerosis are not fully understood, some are multifactorial known to affect the incidence of xerosis, both genetic, environmental, co-morbidity such as hypertension, kidney disorders, and polypharmacy, the severity of xerosis increases with age, as a result the skin barrier function is impaired, with its increasing transepidermal water loss (TWL), decreased skin hydration and sebum levels3,5,6 so that they are prone to infection, ulceration and chronic wounds that are difficult to heal, have a negative impact on the quality of life of the 
elderly. The most common predilection is the extremities, both in the lower limbs, the arms can also be found on the trunk, face, and generalization.7,8 Moisturizer is the first line in the treatment and treatment of xerosis and pruritus in the elderly, which can improve skin barrier function, a moisturizer should have a multifunctional component, as an emollient, occlusive and humectant plus an anti-inflammatory component, protein rejuvenator. 9,10

In 2017, it was found that a moisturizer contains a mixture of plant extracts including calendula, lavender, rosemary, chammomile, vitamins $A$ and $E$ and added with PurCelin Oil. PurCelin oil is an oil ingredient with an olive oil-like consistency, is easily absorbed, is a strong emollient. The components of the plant extract mixture function as anti-microbial, anti-inflammatory, anti-fungal, able to regulate the desquamation process, act as a strong emollient so as to improve skin manifestations. Research on 25 Caucasian women in South Africa with xerosis cutis applied to a mixture of plant extracts on the lower limbs showed significantly improving skin disorders to become smooth visually. ${ }^{11}$ Until now there have been no other studies on the effectiveness of plant extract mixtures

Virgin coconut oil (VCO) is a traditional moisturizer that has been used for a long time in tropical countries, contains lauric, capric, caprrylic and myristic acid, is an emollient and anti-microbial.12,13 Clinical studies compare VCO with mineral oil as a moisturizer, randomization, blindness control in 34 adult patients with mild to moderate xerosis, significantly improving skin hydration and increased surface lipid levels, this study concluded that VCO application was as good as mineral oil. ${ }^{14}$ Another study showed the effect of VCO compared to mineral oil in pediatric patients with Atopic dermatitis, VCO was significantly better than mineral oil in improving skin barrier function, with decreased scoring of atopic dermatitis (SCORAD). ${ }^{12}$

This study aims to prove the effectiveness and safety of the plant extract mixture compared to VCO as a moisturizer for the treatment of xerosis and prurius senilis. In Indonesia this research has never been conducted

\section{Material and Methods}

The research samples were from the Teratai nursing home and the Geriatric clinic of the RSMH Palembang, ethical clearance approval was obtained from the Moh Hoesin Hospital Institute Palembang, and informed consent was obtained from the research subjects. The research was conducted in August-November 2018

Patients: The research subjects were double blinded by randomization of 63 subjects, two subjects were excluded from the study because of death, a total of 61 research subjects followed, namely 36 women and 25 men, with xerosis and senile pruritus. Patients were excluded if they had mental or physical disorders, skin infections / dermatitis and other acute skin diseases and applied antibiotic cream, moisturizers and corticosteroids. Patients were given drugs randomly in 2 treatment groups, the plant extract mixture group consisted of 30 samples and the VCO group consisting of 31 samples,. The composition of the plant extract mixture consists of calendula, lavender, rosemary, chammomile, vitamins $A$ and $E$ plus PurCelin Oil, produced in South Africa and Union Switzerland Ltd imported and distributed in Indonesia PT Radiant Sentral Nutrindo. VCO consists of lauric, capric, caprrylic and myristic acid. VCO is produced in Indonesia. These two oils are put in a dark, odorless bottle, as much as $30 \mathrm{cc} /$ bottle, as many as 30 bottles of plant extract mixture and as many as 31 bottles of $\mathrm{VCO}$

Study design: Research subjects with xerosis and pruritus senile were instructed to use baby soap to clean both legs, not to use other moisturizing creams during the study.

Assessment Groups 1 and 2 were assessed using the Objective dry skin score (ODSS) looking at improvement in objective clinical symptoms, assessment of pruritus using the Visual analog scale (VAS) assessment; Assessment of skin hydration and sebum levels was carried out using a Corneometer (MPA System 6 probe CM 825), and Sebumeter (SM 810 Courage + Khazaka, Germany), respectively. The research subjects explained how to use the lotion on both lower limbs of the volar section of 1 finger tip unit 
(FTU) left and right $2 \mathrm{X} /$ day given randomly. The research subjects were given books to report adverse effects. Researchers conducted a follow-up for the second and fourth weeks, each visit the research subjects were given 1 bottle of either a mixture of plant extracts or VCO. a standard photograph was taken for each visit for documentation

\section{Analysis and Outcome}

The assessment was carried out on the based line, week two and week four by assessing the improvement of the clinical picture with (ODSS), assessment of the severity of pruritus with (VAS), assessment of skin hydration with a chorneometer and sebum levels with sebumeter while assessing side effects with a VAS score. Statistical analysis determined the differences in the group of plant extract mixture compared to VCO on the base line, week 2 and week 4 of treatment, analyzed by Mann-Whitney / Wilcoxon. Statistical analysis of the difference in the effectiveness of the plant extract mixture group compared to VCO at week 2 and week of treatment, analyzed with paired T test, 95\% confidence interval, significant if $(\mathrm{p}<0.005)$ The results of decreasing VAS, ODSS, increase in Corneometer, sebumeter is considered a treatment success. No change or worsening of the VAS, ODSS, Corneometer, sebumeter scores were considered as treatment failure.

\section{Results}

The research subjects were 61 research subjects, divided into 2 treatment groups successively, the plant extract mixture group $70.13 \pm 10.69$; VCO group 72.32 \pm 11.01 The minimum-maximum age range for the plant extract mixture group was 50-97 years and the VCO group 53-98 years. The consecutive mean age of the plant extract mixture group was around $70.13 \pm$ 10.69, the VCO group was around 72.32 \pm 11 .

The results of statistical analysis in the plant extract mixture group compared to the VCO group, the mean VAS score on the base line, week 2 and week 4 with the Wilcoxon test the plant extract mixture group compared to $\mathrm{VCO}$, it was found that there was a significant difference in the mean VAS score (p 0.002). However, in the VCO group, there was a decrease in the mean VAS score, statistically showing no significant mean VAS score (p 0.112).

The results of statistical analysis in the plant extract mixture group compared to the VCO group on the base line, week 2 , week 4 showed the plant extract mixture average ODSS score decreased, the statistical test results showed that there was a significant difference in the mean ODSS score $(\mathrm{p}$ 0.000 While in the VCO group there was no decrease in the mean ODSS score, the statistical test results showed no difference in the mean ODSS score (p 1.00).

The results of statistical analysis of the increase in skin hydration with a corneometer in the plant extract mixture group compared to the VCO group on the base line, week 2, week 4 , in the plant extract mixture group the results of statistical tests showed an increase in the mean skin hudration, indicating that there was a significant difference in the mean corneometer $(\mathrm{p}$ 0.000 ), while the VCO group did not have an increase in the corneometer mean, the statistical test results showed that there was no difference in the mean corneometer ( $\mathrm{p} 0.18$ ).

The results of statistical analysis of the increase in sebum levels with sebumeter, the mixture group of plant extracts compared to VCO on the base line week 2 and week 4 , in the plant extract mixture group the results of statistical tests showed an increase in the mean sebum levels, indicating that there was a difference in the mean sebum ( 0.000 ) while in the VCO group the statistical test results showed no difference in the mean sebum levels (p 0.374). The complete analysis results are presented in table 1 as follows:

The results of statistical analysis, comparison of the effectiveness of the plant extract mxture group compared to $\mathrm{VCO}$ at week 2 and 4 on the assessment of VAS, ODSS, corneometer and sebometer in xerosis and pruritus senile 
The results of the VAS score analysis of the plant extract mixture group compared to the VCO at week 2 and week 4, the plant extract mixture group showed that the VAS score decreased significantly compared to the $\mathrm{VCO}$ group ( $p$ value $=0.000)$

The results of statistical analysis of the ODSS score of the plant extract mixture group compared to the VCO group at week 2 and week 4 showed that the ODSS score in the plant extract mixture group was significantly improved compared to the VCO group ( $p$ value $=0.000$ )
The results of statistical analysis of skin hydration with a corneometer, the plant extract mixture group showed a significant increase compared to the VCO group at week 2 and week 4 ( $\mathrm{p}$ value $=0,000)$.

The results of the analysis of sebum levels with a sebometer, in the plant extract mixture group showed that the sebum level increased significantly compared to the VCO group at week 2 and week 4 ( $p$ value $=0.000)$. The full analysis results are presented in table 2 as follows:

Table 1. Differences in the use of a mixture of plant extracts and VCO on the Assessment of ODSS, VAS, Corneometer and Sebometer at the Baseline, week 2, Week 4

\begin{tabular}{|c|c|c|c|c|c|c|c|c|}
\hline \multirow[b]{2}{*}{ Variable } & \multicolumn{3}{|c|}{ The plant extract mixture } & \multirow{2}{*}{$\underset{\text { value }}{p}$} & \multicolumn{3}{|c|}{ vco } & \multirow[b]{2}{*}{$p$ value } \\
\hline & $\begin{array}{c}\text { Baseline } \\
\overline{\mathbf{x}} \pm \text { SD }\end{array}$ & $\begin{array}{c}\text { Week 2 } \\
\overline{\mathrm{x}} \pm \text { SD }\end{array}$ & $\begin{array}{c}\text { Week } 4 \\
\overline{\mathrm{x}} \pm \text { SD }\end{array}$ & & $\begin{array}{c}\text { Baseline } \\
\overline{\mathbf{x}} \pm \text { SD }\end{array}$ & $\begin{array}{c}\text { Week } 2 \\
\overline{\mathbf{x}} \pm \text { SD }\end{array}$ & $\begin{array}{c}\text { Week } 4 \\
\overline{\mathbf{x}} \pm \text { SD }\end{array}$ & \\
\hline VAS & $4.5 \pm 2.37$ & $2.00 \pm 2.01$ & $0.50 \pm 1.13$ & 0.000 & $5.16 \pm 1.96$ & $4.77 \pm 1.56$ & $4.77 \pm 1.56$ & 0.22 \\
\hline ODSS & $2.5 \pm 0.62$ & $0.86 \pm 0.43$ & $0.26 \pm 0.52$ & 0.000 & $1.61 \pm 0.76$ & $0.74 \pm 0.72$ & $1.58 \pm 0.67$ & 0.782 \\
\hline Corneometer & $22.07 \pm 7.62$ & $32.26 \pm 9.42$ & $40.92 \pm 9.92$ & 0.000 & $19.00 \pm 8.21$ & $19.43 \pm 8.43$ & $19.74 \pm 7.92$ & 0.100 \\
\hline Sebometer & $0.53 \pm 0.68$ & $1.26 \pm 0.86$ & $2.73 \pm 0.98$ & 0.000 & $1.00 \pm 1.23$ & $0.90 \pm 1.13$ & $0.80 \pm 0.98$ & 0.374 \\
\hline
\end{tabular}

Table 2. Comparison of the effectiveness of using plant extract mixtures and VCO on the assessment of ODSS, VAS, Corneometer and Sebometer in elderly xerosis and pruritus Week 2 and Week 4

\begin{tabular}{|c|c|c|c|c|c|c|}
\hline \multirow[b]{2}{*}{ Variable } & \multicolumn{2}{|c|}{ Week 2} & \multirow[b]{2}{*}{$\underset{\text { value }}{p}$} & \multicolumn{2}{|c|}{ Week 4} & \multirow[b]{2}{*}{$\underset{\text { value }}{p}$} \\
\hline & $\begin{array}{c}\text { The plant } \\
\text { extract } \\
\text { mixture } \\
\text { Average } \pm \text { SD }\end{array}$ & $\begin{array}{c}\text { VCO } \\
\text { Average } \pm \text { SD }\end{array}$ & & $\begin{array}{c}\text { Mixture } \\
\text { ekstrak } \\
\text { tumbuhan } \\
\text { Average } \pm \mathrm{SD}\end{array}$ & $\begin{array}{c}\text { VCO } \\
\text { Average } \pm \text { SD }\end{array}$ & \\
\hline VAS & $2.00 \pm 2.01$ & $4.77 \pm 1.56$ & 0.000 & $0.50 \pm 1.13$ & $4.77 \pm 1.56$ & 0.000 \\
\hline ODSS & $0.86 \pm 0.43$ & $0.74 \pm 0.72$ & 0.000 & $0.26 \pm 0.52$ & $1.58 \pm 0.67$ & 0.000 \\
\hline Corneometer & $32.26 \pm 9.42$ & $19.43 \pm 8.43$ & 0.000 & $40.92 \pm 9.92$ & $19.74 \pm 7.92$ & 0.000 \\
\hline Sebometer & $1.26 \pm 086$ & $0.90 \pm 1.13$ & 0.000 & $2.73 \pm 0.98$ & $0.80 \pm 0.74$ & 0.000 \\
\hline
\end{tabular}

\section{Discussion}

Xerosis cutis and pruritus are often found in elderly patients, and are difficult to treat with clinical manifestations in the form of dry, rough, reddish, scaly skin, cracked / cracked skin accompanied by mild to severe pruritus, ${ }^{10,15}$ as a result of an itching-scratching cycle (Itch-scratchitch) causes complications in the form of secondary infection, ulceration and chronic wounds that are difficult to heal.2,16,17 Various moisturizers are widely circulated in the world for skin care. Moisturizer should be efficacious in increasing lipid structure and sebum levels, as well as improving clinical symptoms and manifestations. ${ }^{7}$ Experts recommend that a moisturizer for the treatment and treatment of xerosis with pruritus in the elderly must contain ingredients that are emollient, okluisive, 
humectant that work synergistically, are precise, effective, can be given in the long term, do not cause side effects, make the elderly obedient and comfortable to apply. a mositurizer, and can improve the quality of life. 3,9,17-20 Currently, a moisturizer with a multi-functional composition is found, containing a mixture of essential oils from plant extracts with the composition of calendula, lavender, rosemary, chammomile, vitamins $A$ and $E$ and PurCelin is known as Mixture plant extract $\mathbb{R}$. Mixture of plant extracts has properties as antimicrobial, anti-inflammatory, anti-fungal, as well as a strong emollient, able to improve dry skin. Previous research has shown that plant extract mixtures can improve skin barrier function by reducing TEWL, increasing skin hydration and sebum levels. 11

Virgin coconut oil (VCO), a traditional moisturizer that has long been used as an emollient, has anti-microbial properties. Previous research has been shown to improve the skin barrier function in patients with atopic dermatitis and mild to severe Xerosis. ${ }^{12,21}$

In this study showed that at week 2 the plant extract mixture group and the VCO group with VAS and ODSS examination showed a decrease in pruritus, and clinical improvement, reduction in erythema, scale, fissure, smooth skin texture, had the same effectiveness (p 0,000), but at Week 4 of the plant extract mixture group (p 0.002, p 0.000) showed that VAS and ODSS decreased significantly compared to the VCO group (p 0.112, $\mathrm{p}$ 1.0). With the corneometer and sebumeter in the plant extract mixture group, skin hydration and sebum levels increased significantly (p 0.000) compared to the VCO group (p 0.18, p 0, 68) (table 1). According to previous research, plant extract mixtures have been shown to improve skin barrier function by decreasing TEWL and skin hydration and increasing skin sebum levels, ${ }^{11}$ The essential oil contained in the plant extract mixture consists of lavender, chamomile, rosemary which has an anti-bacterial and anti-pruritic effect, while rosemary and calendula have anti-inflammatory and anti-oxidant effects. pure coconut) contains lauric acid, capric acid, caprrylic acid, oleic acid, palmitic acid, stearic acid, myristic acid as skin barrier protection, antibacterial and also as an emollient. ${ }^{21}$ VCO can improve dry skin, improve skin hydration and increase levels mild to moderate xerosis skin sebum. ${ }^{14}$ According to Evalengista 2014 study in children with atopic dermatitis, VCO was shown to be significantly more effective than mineral oil in reducing pruritus, reducing clinical manifestations with SCORAD assessment, also effective as an antibacterial, especially against Staphylococcos aereus, but not can increase skin sebum levels, 12 pem process VCO production is done traditionally, the content of several enzymes in coconut meat is lost and the risk of contamination with high mycotoxins reduces the effectiveness of $\mathrm{VCO}$, preferably the process of making VCO with high technology.

This study shows that plant extract mixture as a moisturizer has a beneficial effect compared to $\mathrm{VCO}$, Comparative study of the effectiveness of the plant extract mixture group versus VCO; on week 2 and week 4 assessments showed a mixture of plant extracts and VCO, VAS scores, ODSS scores, corneometer, sebumeter there were significant differences after week 2,4 (VAS p0.000, ODSS p 0.000, corneometer p 0.000, sebumeter 0.000 ), whereas there was no significant difference in the VCO group after, 2 and 4 weeks (VAS p 0.112, ODSS 1.00, corneometer 0.18, sebumeter 0.680).

\section{(table 2)}

In one study on 40 healthy female study subjects (20 Caucasians and 20 Negroes) comparing plant extract mixtures with control applied to the lower extremity volar section with a corneometer examination showed that 2 hours after application of plant extract mixtures there was a decrease in transepidermal water loss (TEWL). ) and increased skin hydration compared to controls. Whereas the previous research was a 
randomized, double blind controlled clinical study in 34 adult patients with mild to moderate xerosis comparing VCO with mineral oil as a moisturizer, which significantly increased skin hydration and skin surface lipid levels, this study concluded that VCO application was more effective than mineral oil. ${ }^{14}$ From this research, it is concluded that both of these drugs, both a mixture of plant extracts and VCO can be used as a moisturizer, however, plant extract mixtures are more effective than VCO (see table 2)

Comparison of the effectiveness between the plant extract mixture group and VCO with VAS, ODSS, corneometer and sebumeter showed that the plant extract mixture group was significantly more effective than the VCO group at week 2 and week 4, in improving skin barrier function, increasing skin hydration and sebum levels in The plant extract mixture group increased significantly compared to the VCO group (p 0.000). In the management of xerosis and pruritus, the application of moisturizer as the first line, the components of the moisturizer are multifunctional, namely having occlusive, humectant and emollient properties. decreased size of sebaceous glands and eccrine glands and reduced vascularization with the incidence of xerosis and pruritus senile. 3,7,18 Another study on 25 Caucasian female study subjects applied plant extract mixture compared to untreated healthy skin controls, applied twice to the volar lower leg area, with ODSS, significantly improved clinical manifestations, skin became moist, looked smoother than control.11 In conclusion, plant extract mixtures are effective in improving clinical manifestations. According to previous research Lavender works to inhibit the release of histamine from mast cells so that it acts as an anti-pruritic, Calendula and rosemary have an anti-oxidant effect, while Chamomile has an anti-inflammatory effect. ${ }^{25}$ According to several studies, a mixture of plant essential oils works synergistically, improving Its solubility and bioavabiolity effects, as well as the multitarget pharmacological objectives of each of these essential oils, can also reduce the toxicity and side effects of each essential oil,26 Virgin coconut oil (VCO) is traditionally obtained from fresh cooked coconut meat containing mainly triglycerides mediu mchain. and fatty acids, which function on the skin barrier as a substitute for lost lipids / fats. In addition, VCO contains lauric, capric, caprrylic and myristic acid, as well as anti-septic properties. ${ }^{13}$ This research proves that a mixture of plant extracts as a moisturizer is more effective $\mathrm{d}$ comparable to VCO., but further research is needed for the long term (see table 2)

In this study, observations of adverse effects were assessed by VAS assessment. against the 2 groups Mixture of plant extracts and VCO both at week 2 and 4, no adverse effects were found This study shows that the two groups are well tolerated, Clinical studies comparing VCO with mineral oil as a moisturizer, randomization, double blindness with control in 34 patients Adult mild to moderate xerosis significantly improves skin hydration and increases skin surface lipid levels. This study concludes that VCO application is more effective than mineral oil. 14 In this study, the observation for 4 weeks of the Mixture group of plant extracts and VCO, the research subjects were obedient and comfortable and found no adverse effects until the study was completed, so that the two groups of drugs were safe to use as a moisturizer for xerosis cutis senilis, but in producing $\mathrm{VCO}$ it is necessary sophisticated molecular production techniques to produce potent VCO as moisturizar

\section{Conclusion}

Assessment by ODSS, plant extract mixture showed a significantly better improvement in clinical manifestations than VOC; Assessment by VAS, plant extract mixture was more effective in reducing pruritus significantly than VCO; The plant extract mixture significantly improved 
clinical manifestations, increased skin hydration, sebum levels compared to VCO. The two groups of medicinal plant extract and VCO mixture were not found to have any adverse effects so that they are safe to use as a moisturizer. Further research is needed to assess the effectiveness and compliance of the elderly in using plant extract mixtures

\section{References}

1. Paul C, Maumus-Robert S, MazereeuwHautier J, Guyen CN, Saudez X, Schmitt AM. Prevalence and Risk Factors for Xerosis in the Elderly: A Cross-Sectional Epidemiological Study in Primary Care. Dermatology. 2011; 223: 260-5.

2. Norman RA. Xerosis and pruritus in the elderly: recognition and management. Dermatol Ther. 2003; 16: 254-9.

3. White-chu EF, Reddy M. Dry skin in the elderly: Complexities of a common problem. Clin Dermatol. 2011; 29: 37-42.

4. Haroun MT. Dry Skin in the Elderly. Geriatr Aging. 2003; 6: 41-4.

5. Patel T, Yosipovitch G. Therapy of pruritus. Expert Opin Pharmacother. 2010; 11: 1673-82.

6. Proksch E, Jensen JM. Skin as an Organ of Protection. In: Goldsmith LA, Katz SI, Gilchrest BA, Paller AS, Leffell DJ, Wolf K, editors. Fitzpatrick's Dermatology. 8th ed. New York: McGraw Hill; 2012. p. 486-99.

7. Buraczewska I, Berne B, Lindberg M, Torma H, Loden M.Changes in skin barrier function following long-term treatment with moisturizers, a randomized controlled trial. Br J Dermatol. 2007; 156: 492-8.

8. Berger TG, Shive M, Harper GM. Pruritus in the Older Patient A Clinical Review. JAMA. 2013; 310: 2443-50.

9. Kraft JN, Lynde CW. Moisturizers: What They Are and a Practical Approach to Product Selection. Skin Therapy Lett. 2005; 10: 1-8.
10. Parker J, Scharfbillig $\mathrm{R}$, Jones $\mathrm{S}$. Moisturisers for the treatment of foot xerosis: a systematic review. J Foot Ankle Res. 2017; 10: 1-10.

11. Stephens. Summary: A 12-week clinical study to evaluate the efficacy of Bio-Oil in improving the appearance of aging body skin. 2011.

12. Evangelista MTP, Abad-casintah F, Lopezvillafuerte L. Clinical trial The effect of topical virgin coconut oil on SCORAD index, transepidermal water loss, and skin capacitance in mild to moderate pediatric atopic dermatitis: a randomized, doubleblind, clinical trial. Int J Dermatol. 2014; 53: $100-8$.

13. De las Alas JMG, Carpio VM, Lim MEL, Frez MLF. Randomized Controlled Trial on the Efficacy and Safety of Virgin Coconut Oil Compared to Mineral Oil in the Treatment of Uremic Xerosis. Acta Med Philipp. 2014; 48: 35-42.

14. Agero ALC, Verallo-Rowell VM. A Randomized Double-Blind Controlled Trial Comparing Extra Virgin Coconut Oil with Mineral Oil as a Moisturizer for $M$... Dermatitis. 2016; 15: 109-16.

15. Farage MA, Miller KW, Berardesca E, Maibach HI. Clinical Implications of Aging Skin Cutaneous Disorders in the Elderly. Am J Clin Dermatol. 2009; 10: 73-86.

16. Ganceviciene R, Liakou AI, Theodoridis A, Makrantonaki E, Zouboulis CC. Skin antiaging strategies. Dermatoendocrinol. 2012; 4: 308-19.

17. Garibyan L, Chiou AS, Elmariah SB. Advanced Aging Skin and Itch: Addressing an Unmet Need. Dermatol Ther. 2013; 26: 92-103.

18. Loden $M$. The clinical benefits of moisturizers. JEADV. 2005; 19: 672-88.

19. Surber C, Brandt S, Cozzio A, Kottner J.Principles of Skin Care in the Elderly. Giorn Ital Dermat V. 2015; 150: 699-716. 
20. Yosipovitch G. Dry skin and impairment of barrier function associated with itch - New insights. Int J Cosmet Sci. 2004; 26: 1-7.

21. Lin T, Zhong L, Santiago JL. AntiInflammatory and Skin Barrier Repair Effects of Topical Application of Some Plant Oils. Int J Mol Sci. 2018; 19: 1-21.

22. Ali B, Al-wabel NA, Shams S, Ahamad A, Khan SA, Anwar F. Essential oils used in aromatherapy: A systemic review. Asian Pac J Trop Biomed. 2015; 5: 601-11.

23. Chouhan S, Sharma K, Guleria S. Antimicrobial Activity of Some Essential Oils - Present Status and Future Perspectives. Medicines. 2017; 4: 1-21.

24. Baalham P, Birch I, Young M, Beale C. Xerosis of the feet: a comparative study on the effectiveness of two moisturizers. Br J Community Nurs. 2007; 16: 591-7.

25. Tabassum N, Hamdani M. Plants used to treat skin diseases. Phcog Rev. 2014; 8: 52-60.

26. Orchard A, Vuuren S Van. Commercial Essential Oils as Potential Antimicrobials to Treat Skin Diseases. Evid-Based Complement Altern Med. 2017; 2017: 192. 